\title{
Real-Time Monitoring and Reminding of Remote Peritoneal Dialysis System Based on the Principle of Least Squares
}

\author{
Jia Wu*, Zheng Ji, Min Pi and Tiegang Yi \\ Shenzhen Hospital of Traditional Chinese Medicine, Guangdong Province, Shen Zhen, China
}

*Corresponding author: Jia Wu, Shenzhen Hospital of Traditional Chinese Medicine, Computer center, No 1, Fu Hua Road, 518000, Guangdong Province, Shen Zhen, China, Phone: +86-13826557880; E-mail: 107766353@qq.com

Received: 31 May, 2021 | Accepted: 01 Jul, 2021 | Published: 12 Jul, 2021

Citation: Wu J, Ji Z, Pi M, Yi T (2021) Real-Time Monitoring and Reminding of Remote Peritoneal Dialysis System Based on the Principle of Least Squares. Int J Nephrol Kidney Fail 7(3): dx.doi.org/10.16966/2380-5498.214

Copyright: (C) $2021 \mathrm{Wu}$ J, et al. This is an open-access article distributed under the terms of the Creative Commons Attribution License, which permits unrestricted use, distribution, and reproduction in any medium, provided the original author and source are credited.

\begin{abstract}
Peritoneal dialysis has been widely studied and applied for kidney disease because of its low cost and easy operation. Given the development of chronic kidney disease worldwide, peritoneal dialysis has attracted more and more attention. At the same time, with the development and popularization of mobile network technology, mobile telematics has begun to become a mainstream trend. By integrating the experience of clinicians, the remote diagnosis and treatment system of the peritoneal dialysis developed by Shenzhen Traditional Chinese Medicine Hospital can monitor the entire peritoneal dialysis data of patients. The peritoneal dialysis data were analyzed by statistical methods. In this paper, we designed a data acquisition device with Bluetooth transmission protocol and a user APP to collect peritoneal dialysis data from experimental patients, and built a regression model based on the least square principle according to the clinical data of real patients. Through the model, abnormal or discrete points can be identified in real time. In clinical practice, by analyzing the possible medical risks and adverse events of patients according to the abnormal points, we realize the function of prediction and early reminding. The system indicates the results to patients according to the confidence interval of regression prediction, which greatly strengthens the interaction of the system and improves patient compliance.
\end{abstract}

Keywords: Remote peritoneal dialysis system; Least square method; CAPD; Filtration rate per minute

\section{Introduction}

The prevalence of long-term dialysis is increasing worldwide [1]. In recent years, a concept of renal rehabilitation has been widely known among nephrology specialists, dialysis specialists, kidney transplantation specialists, rehabilitation specialists, nutrition specialists, guideline specialists, nurses, physiotherapists, and representatives of patients [2]. A large proportion of patients with renal failure are treated by continuous ambulatory peritoneal dialysis (CAPD), and its role in renal replacement programs has been more clearly defined now, with steadily improved technique and survival in patients [3]. Peritoneal dialysis has been highly valued by the medical industry, which has also been actively promoted by the government as treatment programs. The exciting mushrooming of scientific knowledge on various aspects of peritoneal dialysis has led to a better understanding of the technique [4].

Peritoneal dialysis is one of the most crucial methods for renal replacement therapy, typically at home, with largely restricted efficacy by patients' normative and follow-up regularity. Poor patient compliance to the therapy leads to worsening conditions, often increasing healthcare costs [5]. However, because of the lack of followup data, there are some defects in the current peritoneal dialysis follow-up model, which makes it difficult to correct the patients' nonstandard operation in time.
With the improvement of computer technology and the application level of medical information, the construction of "Medical + Internet" mode provides a feasible solution for follow-up diagnosis and treatment of certain diseases in remote areas [6]. On this basis, telemedicine has been widely used in medical care that is good news for patients living in remote communities and areas, which not only can provide patients with relevant information and services [7], but also can support patients living in remote areas and help them in maintaining independent status while inculcating a greater degree of confidence that well-trained professionals are closely monitoring the therapy and are readily available for assistance, with a clinical benefit $[8,9]$.

At present, various remote systems have been developed to assist in peritoneal dialysis. Caogen Hong managed peritoneal dialysis in remote areas with the information management system. Moreover, he established a peritoneal dialysis remote management system, including medical client and medical and patient applications, which replaced the telephone center manual recording and the old follow-up management mode through telephone [10]. Gallar P, et al., conducted a medical conversation session through a video conferencing device installed in each patient's home [11]. Cai Y, et al., controlled and provided real-time guidance through remote monitoring and management, thus reducing the chance of contact infection in patients 
undergoing peritoneal dialysis. Thus, patients in remote areas can be given better care through the video surveillance technology for the management of peritoneal dialysis patients in remote areas combined with the knowledge of nursing for the management of peritoneal dialysis [12]. Nayak KS, et al., developed an application for tablet computers as an interactive user guide to support patients, making peritoneal dialysis more accessible. Moreover, they taught patients with tablet computers, showing complete training, and providing feedback on the operation to patients. The Columbian Remote Automated Peritoneal Dialysis System, used by Alfonso Bunch, provides two-way communication between patients and clinical teams by introducing the remote patient monitoring (RPM), which significantly improves patient compliance and treatment effects on patients by evaluating its increasing use $[13,14]$.

Although the past remote peritoneal systems improved the interaction between doctors and patients and enhanced the efficacy through various methods, they were just tools for remote conversations, which rarely mentioned how to improve their quality with the data of remote diagnosis and treatment. Despite their effective online communication and interaction, they do not ensure long-term stability. The human interaction monitoring model requires active and patient service of medical personnel, but long-term observations can cause fatigue, and some minor differences in peritoneal dialysis are difficult to find sometimes. To improve the follow-up effect on patients with CAPD at home, to reduce the risk of complications from irregular follow-up, and to improve clinical efficacy and quality of medical services effectively, a telediagnosis and treatment system for peritoneal dialysis was developed by the medical personnel from the Nephrology Department of Shenzhen Hospital of Traditional Chinese Medicine in conjunction with Information Technology Engineer team. The system includes two parts: backstage management of computer and mobile application. By integrating the Internet of things technology, the data of monitoring indicators, such as daily dialysis ultrafiltration volume and body weight change of home CAPD, can be collected. The abnormality of peritoneal dialysis can be monitored in one aspect by determining the historical peritoneal data of patients through the least-squares method.

\section{Methods}

\section{Introduction to data acquisition and operation of peritoneal dialysis remote system}

In our hospital, the data from remote diagnosis and treatment system of peritoneal dialysis were provided to medical personnel on a timely basis by combining the Internet of things and peritoneal treatment. Medical personnel supervised the entire treatment process and solved the shortcomings of current CAPD treatment. The peritoneal dialysate was obtained after weighing with a Bluetooth device during the dialysis. Then, the data of peritoneal dialysate (weight, dialysis time, type, and identity of patients undergoing dialysis) were transmitted to the real-time background system for peritoneal dialysis through the mobile network. In addition, medical personnel managed patients' information from several aspects, including patient file management, information management of peritoneal dialysis treatment, peritoneal dialysis management, and follow-up management.

\section{Patients and data}

The Medical Ethics Committee of Shenzhen Hospital of Traditional Chinese Medicine approved the study, approval number: B2019043. A total of 36 patients aged 20-68 years old undergoing peritoneal dialysis in the Nephrology Department of our hospital from January 2017 to June 2019 were enrolled in this study, including 15 males and 21 females. All the patients were treated with Baxter peritoneal dialysate. Peritoneal dialysis data selected in this study were from patients receiving peritoneal dialysate with calcium concentration of $1.5 \%$. The following data were obtained from the peritoneal dialysis system: duration of peritoneal dialysis ( $t$ ), inflow weight (in-weight), and effluent weight (off-weight). The filtration rate per minute $v t$ was calculated according to the available data. The duration of peritoneal dialysis $(\mathrm{t})$ was equal to the time point of peritoneal dialysate minus the time point of infusion (unit: minute). Considering the patient compliance and regularity of peritoneal dialysis operation, peritoneal dialysis within $180 \sim 600 \mathrm{~min}$ of operation time was considered effective, and that without meeting this time range was considered invalid. The quality control standard range of Baxter peritoneal dialysate was $2.2 \pm$ $0.08 \mathrm{~kg}$. When the fluid inflow weight uploaded by patients was out of this range, the operation was considered to be incorrect, which did not conform to the specifications, which were excluded from this study. The filtration rate per minute $v_{t}$ was equal to the fluid output divided by the peritoneal time. The patients' original data were obtained from the tele diagnosis and treatment system of peritoneal dialysis in Shenzhen Chinese Medicine Hospital. After processing, 20,608 valid data meeting the standards are shown in table 1 as below.

The data of abdominal communication were collected in the system database through transformed Internet of things electronic scale equipment with Bluetooth transmission protocol as the carrier. All the patients were informed of the experiment and signed an informed consent, they were also free to evacuate at any time, which would not affect conventional treatment. The patients' identity data of the diagnosis and treatment were uploaded into the system by the nursing staff and maintained regularly, which was free for patients to choose.

\section{Least square regression for peritoneal dialysis data}

The effluent weight, filtration rate per minute, and duration of peritoneal dialysis were calculated for patients with CAPD according to the data uploaded in the peritoneal system. As the value of filtration rate per minute was extremely small, the filtration rate was increased by a factor of 1000 in order to observe the relationship between the data.

Table 1: Descriptive statistics of filtration rate and peritoneal time per minute.

\begin{tabular}{|c|c|c|c|c|c|c|c|}
\hline & \multirow{2}{*}{$\begin{array}{c}\text { Range } \\
\text { Statistics }\end{array}$} & \multirow{2}{*}{$\begin{array}{c}\text { Minimum value } \\
\text { Statistics }\end{array}$} & \multirow{2}{*}{$\begin{array}{c}\text { Maximum value } \\
\text { Statistics }\end{array}$} & \multicolumn{2}{|c|}{ Average value } & \multirow{2}{*}{$\begin{array}{c}\text { Standard deviation } \\
\text { Statistics }\end{array}$} & \multirow{2}{*}{$\begin{array}{l}\text { Variance } \\
\text { Statistics }\end{array}$} \\
\hline & & & & Statistics & Standard error & & \\
\hline $\begin{array}{l}\text { Filtration rate per } \\
\text { minute }(\mathrm{kg} / \mathrm{min})\end{array}$ & 1.83 & 0.96 & 2.78 & 1.8508 & 0.00216 & 0.30999 & 0.096 \\
\hline $\begin{array}{l}\text { Duration of peritoneal } \\
\text { dialysis (min) }\end{array}$ & 420 & 180 & 600 & 367.96 & 0.748 & 107.374 & 11529.210 \\
\hline
\end{tabular}


A scatter plot was drawn by placing the duration of peritoneal dialysis (unit: minute) on $\mathrm{x}$-axis and the filtration rate per minute (expanded 1000 times) on y-axis. Data of a patient were randomly selected with a linear regression function. The regression of $v_{t}$ and $t$ was obtained with the least square method, wherein $v_{t}$ denotes the filtration rate per minute (multiplied by 1000 times) and $t$ the peritoneal time:

$$
v_{t}=-0.017 \mathrm{t}+13.107 \mathrm{R}^{2}=0.9014 \text { (Formula } 1 \text { ) }
$$

The relationship between $v_{t}$ and $\mathrm{t}$ was well displayed in the aforementioned formula. As noted, $\mathrm{R}^{2}=0.9$ has a high overall fitness, and the graph is shown below in figure 1 .

According to figure 1, the filtration rate per minute was associated with time. Pearson's correlation coefficients were calculated by SPSS software. The results of 20,608 valid data are shown in table 2 as below.

The filtration rate per minute decreased with the increase of time. However, with the increase of time, the decline rate was slow, and the curve was approximately a power function. Pearson's correlation coefficient of filtration rate per minute and time was -0.939 , as shown in table 2, with a statistically significant strong negative correlation between them.

There was also a significant difference between fitted curve and actual value in the partial interval range. However, the model could not depict the changing trend between $v_{t}$ and $\mathrm{t}$. The linear regression function model can be improved further. Considering that the actual curve is in the shape of a power function, it is assumed as follows:

$$
v_{t}=a t^{b}
$$

where, $\mathrm{a}$ and $\mathrm{b}$ are unknown parameters, $\mathrm{t}$ is the duration of peritoneal dialysis, and $v_{t}$ is the filtration rate per minute, where, $\mathrm{t}$ indicates the peritoneal time. After performing logarithm transformation on both sides of the formula, we can obtain as below:

$$
\ln v_{t}=\ln a+b \ln t
$$

let $v_{t}^{*}=\ln v_{t}, a^{*}=\ln a, t^{*}=\ln t$; thus, a linear relationship between $v_{t}^{*}$ and $t^{*}$ can be obtained as below:

$$
v_{t}^{*}=a^{*}+b t^{*} \text { (Formula 2) }
$$

Considering the selected data as an example in the same manner, after $v_{t}$ and $\mathrm{t}$ are logarithmized, the scatter plot of the obtained data is shown as below in figure 2 .

\section{Data quality control methods}

As the least square method used in the regression analysis, interval estimation was performed for predicting the results. The confidence interval range of regression was calculated by setting the confidence interval. When the patient's final uploaded data were out of the confidence interval, the patient and medical personnel were reminded through the peritoneal system that the data might exceed or fall below the normal data range. The statistical methods used are as follows:

Let the regression equation calculated according to the least-squares method as follows:

\section{$\hat{Y}=\mathrm{kX}+\mathrm{b}$}

where, $\hat{Y}$ is the predicted value of dependent variable $\mathrm{Y}, \mathrm{X}$ is the independent variable, and $\mathrm{k}$ and $\mathrm{b}$ are estimated values of the regression. An estimate of the model was obtained with the linear regression equation of the sample, with a certain error between predicted and actual values. Therefore, providing a confidence interval for the prediction at a certain neutral level can make our predictions better targeted.

$$
\hat{Y}-Y \sim N\left\{0,\left[1+\frac{1}{\tau}+\frac{\hat{(X}-\bar{X})^{2}}{\Sigma\left(X_{i}-\bar{X}\right)^{2}}\right] \sigma^{2}\right\}(\text { Formula } 3)
$$

where, $\hat{X}$ represents the independent variable to be predicted, $\hat{Y}$ corresponds to the predicted value $\hat{X}$ calculated by the regression equation, and $\sigma^{2}$ represents the total variance and which can be replaced by the sample variance:

$$
\sigma^{2} s^{2}=\left(\sum \hat{u}_{t}^{2} /(T-2)\right)=\sum(\hat{Y}-Y)^{2} /(T-2) \mathrm{T} \text { is the number }
$$
of samples. According to equation 1, the $t$ statistic can be constructed to obtain a prediction interval with a confidence level of 1- $\alpha$ :

$\left[\hat{Y}-\operatorname{tg} \frac{\underline{\alpha}}{2} \times \hat{\sigma} \sqrt{1+\frac{1}{\tau}+\frac{\hat{(X}-\bar{X})^{2}}{\sum\left(X_{i}-\bar{X}\right)^{2}}}, \hat{Y}+\operatorname{tg} \frac{\underline{a}}{2} \times \hat{\sigma} \sqrt{1+\frac{1}{\tau}+\frac{(\hat{X}-\bar{X})^{2}}{\sum\left(X_{i}-\bar{X}\right)^{2}}}\right]$ (Formula 4)

The statistic $\frac{t_{a}}{2} \mathrm{n}$ the above formula can be obtained according to the look-up statistical table. And all the other variables can be calculated. Therefore, a certain deviation range can be set with the above formula

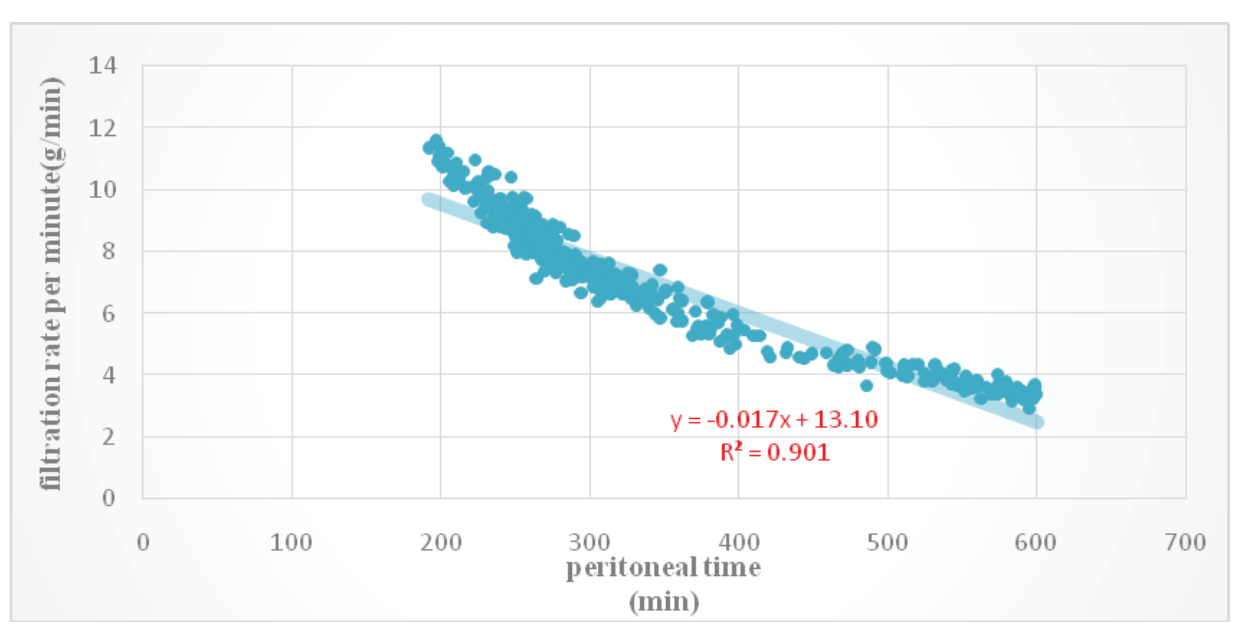

Figure 1: Scatter plot of filtration rate per minute and duration of peritoneal dialysis (the data came from a randomly selected patient). 
Table 2: Correlation between filtration rate per minute and duration of peritoneal dialysis.

\begin{tabular}{|l|l|c|c|}
\hline \multicolumn{2}{|c|}{} & Filtration rate per minute & \multicolumn{1}{c|}{ Duration of peritoneal dialysis } \\
\hline \multirow{2}{*}{ Filtration rate per minute } & Pearson's correlation & - & $-.939 * *$ \\
\cline { 2 - 4 } & Significant (two-tailed) & 0.000 \\
\hline \multirow{2}{*}{$\begin{array}{l}\text { Duration of peritoneal } \\
\text { dialysis }\end{array}$} & Pearson's correlation & $-.939 * *$ & 1 \\
\cline { 2 - 4 } & Significant (two-tailed) & 0.000 & - \\
\hline
\end{tabular}

**At 0.01 level (double tails), the correlation was significant.

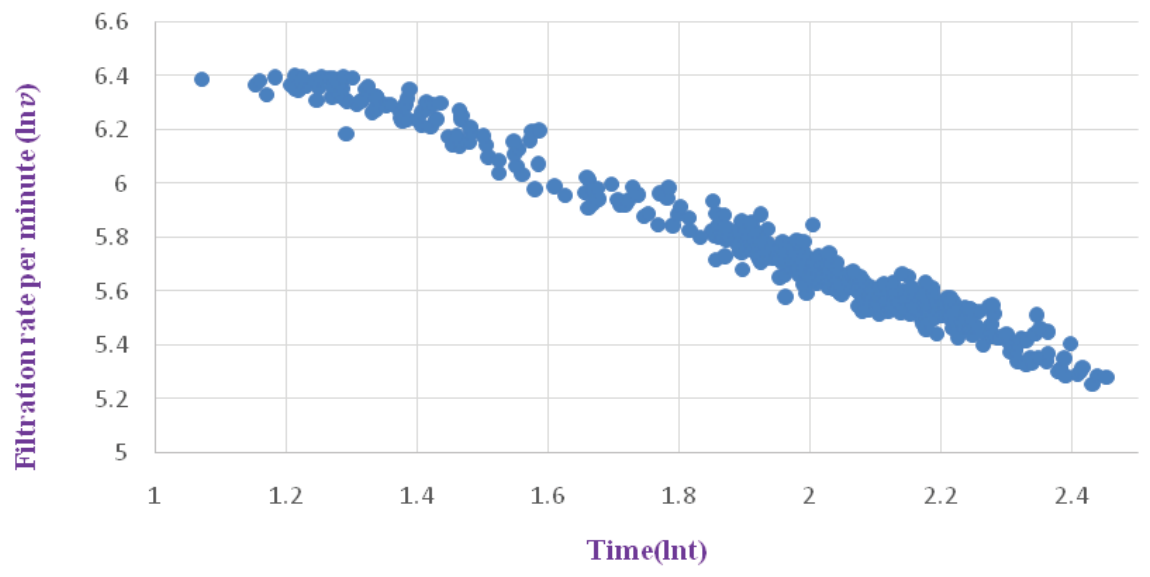

Figure 2: Scatter plot of logarithmic transformation of filtration rate per minute and duration of peritoneal dialysis (the data came from the selected patient in figure 1).

and the expected confidence. When the actual dialysate output data of peritoneal dialysis exceeds the predicted interval, the relevant personnel will be reminded to check for leakage of peritoneal flow. In fact, only formula 4 can predict reasonable intervals. Statisticians have shown that the formula is reliable, with all the variables calculated, which needs to be applied in the diagnosis of peritoneal dialysis.

\section{Results}

\section{Regression results}

A logarithmic transformation was applied to peritoneal time and filtration rate. Linear regression was performed on the entire data with SPSS software version 24.0, with the results evaluated, as shown in table 3 and table 4 below:

The regression function is expressed as follows:

$$
\ln v_{t}=-1.085 \ln t+8.218 \mathrm{R}^{2}=0.953 \text { (Formula 5) }
$$

where, $\mathrm{R}^{2}$ is equal to 0.95 , and the overall fitness of the equation is extremely high. As only one variable was observed in this regression model, the coefficient of regression equation was not zero according to $F$ value obtained from table 3 . According to the t-statistic of coefficient table, constant term and independent coefficient value were not zero. The change of independent variables had an effect on dependent variables. Therefore, a linear regression function appeared.

According to the results, the duration of peritoneal dialysis was a crucial explanatory variable of the filtration rate per minute, and the logarithm of filtration rate per minute and duration of peritoneal dialysis was linear. That is, when other conditions remain unchanged, the logarithm of duration of peritoneal dialysis increased by one unit, and that of filtration rate per minute decreased by 1.085 units. For the applicability of formula 5 , formula 6 is available as below:

$$
v_{t}=3707.1 * t^{-1.085} \text { (Formula 6) }
$$

Formula 6 shows a power function relationship where duration of peritoneal dialysis was an independent variable, and filtration rate per minute was a dependent variable. Let $M_{t}$ be the volume of effluent at the duration of peritoneal dialysis $t$, thus:

$$
M_{t}=V_{t} * t \text { (Formula 7) }
$$

Formula 6 can be substituted into formula 8 , as below:

$$
M_{t}=3707.1 * t^{-0.085} \text { (Formula 8) }
$$

\section{Quality control results}

In this study, the logarithm of filtration rate per minute of peritoneal dialysate and peritoneal time was regressed with the least-squares regression method. However, this study emphasized the clinical practical value of reviewing the results of equations. Furthermore, the weight of peritoneal dialysate can be predicted on the basis of historical data in the future, which is not widely used in the actual treatment. However, comparing the difference between the predicted value and the actual value could help us to know whether the real-time operation of home-based peritoneal dialysis was standard, which had more realistic significance for remote diagnosis and treatment. The time-to-time calibration of abdominal effusion volume errors with statistical methods can be achieved, as shown in figure 3. 
Table 3: Analysis of variance.

\begin{tabular}{|l|c|c|c|c|}
\hline \multicolumn{1}{|c|}{ Model } & Sum of Squares & df & Mean Square & Sig. \\
\hline Regression & 1886.886 & 1 & 1886.886 & 416882.771 \\
\hline Residual & 93.266 & 20606 & $0.000^{\text {b }}$ & 0.005 \\
\hline Total & 1980.152 & 20607 & & \\
\hline
\end{tabular}

a. Dependent Variable: Filtration rate per minute (InVt).

b. Predictors: (Constant), duration of peritoneal dialysis (Int).

Table 4: Regression coefficients and t statistics.

\begin{tabular}{|l|c|c|c|c|}
\hline \multirow{2}{*}{ Model } & \multicolumn{2}{|c|}{ Unstandardized Coefficients } & Standardized Coefficients & Sig. \\
\cline { 2 - 5 } & B & Std. Error & - \\
\hline Constant & 8.218 & 0.010 & -0.000 \\
\hline Duration of peritoneal dialysis (Int) & -1.085 & 0.002 & -.976 & -645.665 \\
\hline
\end{tabular}

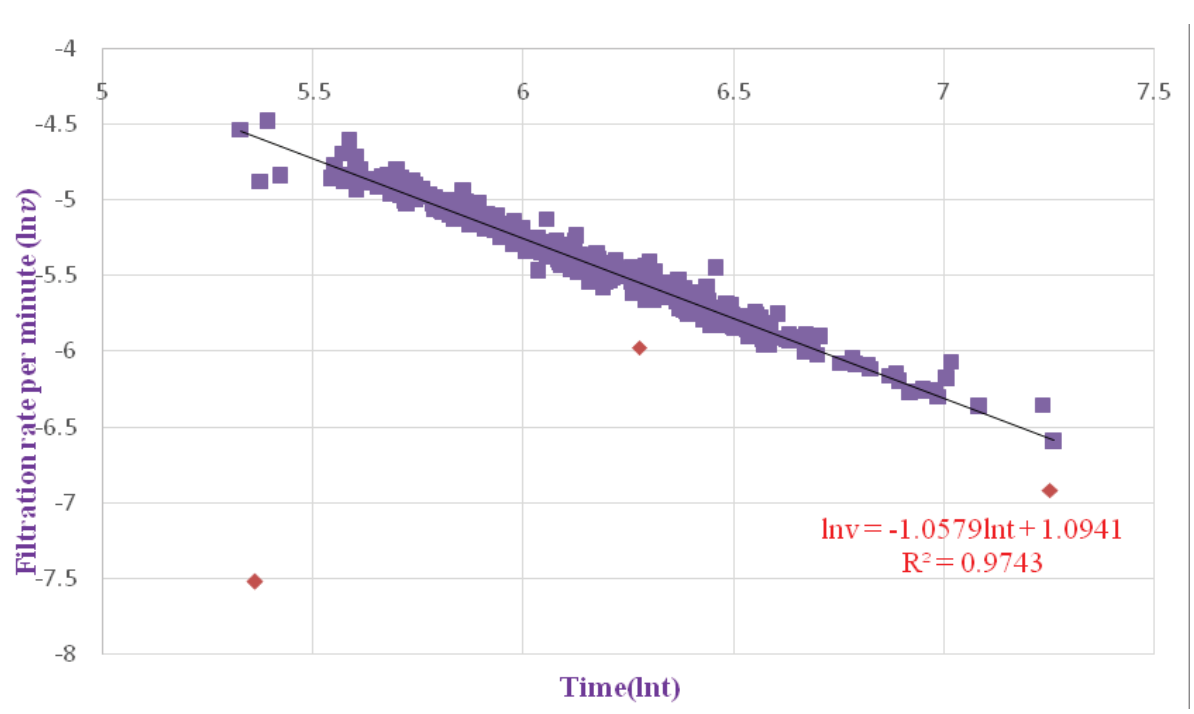

Figure 3: Evaluation of outlier markers; Dots marked in orange are abnormal points (the data come from a randomly selected patient).

Figure 3 is a scatter plot of logarithm of filtration rate per minute (v) and logarithm $(\mathrm{t})$ of duration of peritoneal dialysis from a patient in our hospital. The confidence level was $95 \%$. During the peritoneal dialysis, the prediction interval was calculated according to the regression equation from the previous data. When the final data uploaded by the patient are greater than the maximum value of prediction interval, the change point is marked as orange-red. When the actual point is less than the minimum value of prediction interval, it is marked as green, and the other normal points are marked as yellow. Figure 3 shows that the patient had three low and none high filtration rates of peritoneal dialysis. This model can be used in the peritoneal dialysis system for providing immediate reminders. Patients can easily review the previous operation steps, and medical personnel can reduce some pressure and concentrate more on other aspects.

\section{Analytical Results and Discussion}

The peritoneal dialysis system sets a certain error range in the background. By comparing the calculated value of peritoneal dialysis and the actual uploaded data, any problem in the diagnosis and treatment of patients can be detected if the output volume exceeds the error range, which can also provide a possible basis for the establishment of prevention and control points of corresponding clinical events. At the same time, suggestions for improvement of patients' treatment plan were recommended according to specific situations, and the effective online and offline interactive follow-up mechanism and the complication risk prevention and control model were established to alleviate patients' pain pressure and improve treatment effects.

If the value exceeds the confidence interval, there will be problems in the peritoneal dialysis. The main cause can be found in time according to specific situations. However, there is no guarantee that no problem exists with the peritoneal dialysis for the points meeting the confidence interval. Further exploration is still needed in the future. In addition, according to the treatment plan of peritoneal dialysis patients, their clinical treatment was mainly conducted with $1.5 \%$ Baxter peritoneal dialysate. Therefore, the experimental data used in 
this paper were also based on $1.5 \%$ Baxter peritoneal dialysate. For other scenarios, we need conduct further research in the future.

\section{Conclusions}

In summary, the development of peritoneal dialysis system has promoted the standardized collection of peritoneal data and provided an efficient method for large-scale data census and analysis. However, in the past, remote diagnosis and treatment systems were typically for recording data and providing remote real-time video, audio, image carriers, etc. Few systems achieved the functions of automatic decisionmaking machine with data calculation. By analyzing and processing peritoneal dialysis data, medical personnel could reduce medical risks caused by complications, improve work efficiency, as well as treatment effect, and patients could also benefit a lot from it. The use of regression equations for interval estimation of peritoneal dialysate volume had a significant clinical significance. In order to study the peritoneal dialysis data, the accuracy of peritoneal data prediction has been improved and the application of peritoneal data has been broadened by continuous optimization and improvement of the model. The development and application of peritoneal dialysis system aim to improve peritoneal medical services, identify the real-time transmission of treatment data, promote communication between doctors and patients, and improve the mode of follow-up, so as to improve the curative effect. Moreover, with its development, the integrity and reliability of peritoneal data are guaranteed, greatly reducing the risk of data loss and omission and providing strong support for diagnosis and treatment. In this study, the regression function between the filtration rate per minute of peritoneal dialysate and duration of peritoneal dialysis can provide a certain basis for medical personnel to judge the effect of peritoneal dialysis. Our goal is to help in spreading awareness about the automatic diagnosis and treatment, relying on data from the telemedicine system. Although there are still some shortcomings and limitations in its use, we hope to promote the intelligent management of data, rather than just consider the remote system as a tool without thinking.

\section{Disclosure Statement}

All the authors declared no conflicts of interests and no funding sources.

\section{Acknowledgement}

This work was supported by grant of no. B2019043 from the Guangdong Medical Science and Technology Research Fund Project, China.

The authors thanks the team members from the Computer Center of Shenzhen Traditional Chinese Medicine Hospital for participating in the system construction, the Nephrology Department of Shenzhen Traditional Chinese Medicine Hospital for participating in the collaborative peritoneal dialysis team, and the Jirong Zhong team from
Shenzhen City Hidohear Technology Ltd. for their technical support and help in the project development.

\section{References}

1. Cho Y, Bello AK, Levin A, Lunney M, Osman MA, et al. (2021) Peritoneal Dialysis Use and Practice Patterns: An International Survey Study. Am J Kidney Dis 77: 315-325.

2. Yamagata K, Hoshino J, Sugiyama H, Hanafusa N, Shibagaki $Y$, et al. (2019) Clinical practice guideline for renal rehabilitation: systematic reviews and recommendations of exercise therapies in patients with kidney diseases. Renal Replacement Therapy.

3. Lin J, Ye H, Yi C, Li J, Yu X, et al. (2020) The negative impact of depressive symptoms on patient and technique survival in peritoneal dialysis: a prospective cohort study. Int Urol Nephrol 52: 2393-2401.

4. Jiang J, Wang LH, Fei YY, Zhou XW, Peng L, et al. (2018) Serum Albumin at Start of Peritoneal Dialysis Predicts Long-Term Outcomes in Anhui Han Patients on Continuous Ambulatory Peritoneal Dialysis: A Retrospective Cohort Study. Kidney Dis (Basel) 4: 262-268.

5. Zhang Y, Chen M, Leung VCM, Lai RX (2017) Topical Collection on "Smart and Interactive Healthcare Systems". J Med Syst 41: 121.

6. Lai R, Liu H (2018) Design of network-based intelligent peritoneal dialysis system. Transducer and Microsystem Technologies.

7. Moncrief JW, Popovich RP, Okutan M, Decherd JF (1976) A model of the peritoneal dialysis system. Proc $25^{\text {th }}$ Annu Cong Eng Med Biol 14: 142.

8. Ing TS, Quon MJ, Daugirdas JT, Gandhi VC, Epstein MB (1981) Preparation of bicarbonate containing peritoneal dialysate using an automated dialysate delivery system. Int J Artif Organs 4: 148-149.

9. Nayak KS, Ronco C, Karopadi AN, Rosner MH (2016) Telemedicine and remote monitoring: supporting the patient on peritoneal dialysis. Perit Dial Int 36: 362-366.

10. Hong C (2017) Design and implementation of remote management system for peritoneal dialysis. Automation Application 06: 51-52.

11. Gallar P, Gutierrez M, Ortega O, Rodriguez I, Oliet A, et al. (2006) Telemedicine and follow up of peritoneal dialysis patients. Nefrologia 26: 365-371.

12. Cai Y, Zhang $Y$ (2018) Interventional Study of Remote Technical Guidance for Patients with Peritoneal Dialysis at Home. Hospital Management Forum 35: 73-74.

13. Karopadi AN, Antony S, Subhramanyam SV, Nayak KS (2013) Remote monitoring of peritoneal dialysis: Why? Where? How? Hong Kong J Nephrol 15: 6-13.

14. Bunch A, Vesga JI, Camargo DO, Corzo L, Molano AP, et al. (2019) Remote Automated Peritoneal Dialysis Management in Colombia. Kidney Int Rep 4: 873-876. 